\title{
I. Biogenesis of metalloproteins
}

\section{I-01}

\section{Traversing the ferritin iron channel to mineral and back}

Elizabeth C. Theil ${ }^{1,2}$, Xiaofeng Liu ${ }^{1}$, Takehiko Tosha ${ }^{1}$

${ }^{1}$ Council on Bioiron at CHORI (Children's Hosp. Oakland Res. Inst.), 5700 Martin Luther King Jr. Way, Oakland, CA 94609, USA.

${ }^{2}$ Department of Nutritional Science and Toxicology, University of California, Berkeley, CA 94720, USA. etheil@chori.org

Ferritin protein nanocages, self-assembled from 12 or 24 polypeptide subunits, contain four structural features required for the reversible concentration of iron as hydrated ferric oxide nanominerals: (1) External, gated iron entry/exit pores. (2) Catalytic, oxidoreductase sites that couple $2 \mathrm{Fe}^{2+}$ with $\mathrm{O}_{2}$ to form mineral precursors. (3) A large cavity $(5-8 \mathrm{~nm})$ in the center of the cage where the mineral forms. (4) Channels that connect the pores, catalytic sites with exits into the cavity. Variable numbers of iron occur in the ferritin minerals with up to several thousand iron atoms in the 24 subunit ferritins. Primary sequences can vary $80 \%$ among ferritins, but quaternary cage structure, secondary structure, $4-\alpha$-helix bundles, and pores are conserved. Recent collaborative studies with P. Turano and I. Bertini, L. Patterson and M.L. Miller, J. Schwartz and E.I. Solomon, identify peptide-chelator interactions with the exterior gated pores, the channel, the mid-channel catalytic sites and channel exit pores into the cavity. How entering hydrated $\mathrm{Fe}^{2+}$ is converted to ferric mineral in the cavity and possible mechanisms for reducing the mineral to $\mathrm{Fe}^{2+}$ that exits at the gated pores will be described to illustrate the activities related to diiron oxygenases, ion channel proteins and aquaporins that are combined in ferritins.

Part support NIH grant DK20251 (ECT, XL, TT) and JSPS (TT).

\section{I-02}

\section{NMR of large molecular assemblies: focusing on iron metabolism}

Paola Turano

CERM, University of Florence, Florence, Italy. turano@cerm.unifi.it Besides its use as a structural method, NMR spectroscopy is unique in its capability to reveal, at atomic resolution, macromolecular interactions, conformational changes, and dynamics. Advances in spectroscopic and isotope labeling methods have expanded the range of NMR-affordable biochemical problems. Here, examples are provided of the use of new NMR approaches to obtain valuable information on high molecular weight systems involved in the biology of iron.
I-03

The multi-ligand sensor in the large-conductance $\mathrm{Ca}^{2+}$ - and voltage-gated Slo1 $\mathrm{K}^{+}$channel is essential for its carbon monoxide sensing

Shangwei Hou ${ }^{1}$, Frank Horrigan ${ }^{2}$, Stefan Heinemann ${ }^{3}$, Toshinori Hoshi ${ }^{1}$

${ }^{1}$ Department of Physiology, University of Pennsylvania, Philadelphia, PA 19104, USA.

${ }^{2}$ Department of Molecular Physiology and Biophysics, Baylor College of Medicine, Houston, TX 77030, USA.

${ }^{3}$ Lehrstuhl für Biophysik, Friedrich-Schiller-Universität Jena, 07745 Jena, Germany. hoshi@hoshi.org

Large-conductance $\mathrm{Ca}^{2+}$ - and voltage-gated $\mathrm{K}^{+}$(Slo1 BK) channels are tetrameric transmembrane $\mathrm{K}^{+}$-selective ion channel proteins opened by an increase in intracellular $\mathrm{Ca}^{2+}$ and/or plasma membrane depolarization, and contribute to a variety of physiological phenomena. The physiological versatility of the channel is in part conferred by its allosteric gating mechanism encompassing concerted opening and closing of the ion conduction gate, activation of transmembrane voltage sensors and binding of multiple ligands to the cytoplasmic sensors. The gating machinery function is directly modulated by a variety of physiological signaling molecules including free heme/hemin, carbon monoxide and $\mathrm{H}^{+}$. For example, nanomolar concentrations of free heme/hemin acutely regulate the channel activity in part by weakening the strength of allosteric coupling between the channel's gate and voltage sensors. In contrast, carbon monoxide and $\mathrm{H}^{+}$promote channel opening by partially mimicking the action of intracellular $\mathrm{Ca}^{2+}$. The $\mathrm{Ca}^{2+}$-dependent activation of the Slo1 BK channel is typically considered to involve two distinct $\mathrm{Ca}^{2+}$ sensors: RCK1 sensor and $\mathrm{Ca}^{2+}$ bowl sensor. The stimulatory effects of carbon monoxide and $\mathrm{H}^{+}$are dependent on the RCK1 $\mathrm{Ca}^{2+}$ sensor. In particular, two His residues located in the $\mathrm{RCK} 1 \mathrm{Ca}^{2+}$ sensor are essential. Activation of the channel by $\mathrm{H}^{+}$may involve movement of the aforementioned two His residues by several angstroms. We postulate that the $\mathrm{RCK} 1 \mathrm{Ca}^{2+}$ sensor is a multi-ligand sensor capable of detecting $\mathrm{Ca}^{2+}$, carbon monoxide and $\mathrm{H}^{+}$.

\section{I-04}

Cytochrome $c$ assembly: ever increasing variation and mystery

Stuart J. Ferguson, Alan D. Goddard, James W.A. Allen, Julie M, Stevens.

Department of Biochemistry, University of Oxford, South Parks Road, Oxford OX1 3QU, UK. ferguson@bioch.ox.ac.uk

Formation of cytochromes $c$ requires a deceptively simple posttranslational modification, the formation of two thioether bonds (or 
rarely one) between the thiol groups of two cysteine residues found in a $\mathrm{CXXCH}$ motif (with some occasional variations) and the initial vinyl groups of heme. It has emerged that there are three partial characterised systems for doing this; within these systems there is also variation. Particularly, dramatic is a recent finding that a novel, and believed essential histidine to heme bond in the CcmE protein of System I is not present in a variant form of System I. In addition, there are clear implications for two other distinct systems. Here, some of the current issues in understanding these systems are analysed.

\section{I-05}

\section{The role of heme chaperone protein, $\mathrm{CcmE}$,} in maturation of cytochrome $c$

Takeshi Uchida, Ryoki Kawamura, Koichiro Ishimori

${ }^{1}$ Department of Chemistry, Graduate School of Science, Hokkaido

University, Sapporo 060-0810, Japan. uchida@sci.hokudai.ac.jp

$c$-type cytochromes are electron transfer proteins that are essential for the life of almost all organisms. They characteristically have covalently bound heme via thioether bonds to two cysteines in the protein. In Gram-negative bacteria, biogenesis of $c$-type cytochrome is conducted by a multiprotein complex system known as a cytochrome $c$ maturation $(\mathrm{Ccm})$ system. This system consists of eight gene products $(\mathrm{ccmA-}$ $c c m H$ ). CcmE, which binds heme and delivers it to apocytochrome $c$, is called as a heme chaperone. In the previous study, covalent attachment of heme to cytochrome $c$ was achieved in the presence of only $\mathrm{CcmE}$, heme, and dithiothreitol [1]. However, the precise mechanism remains to be clarified. In this study, we applied UV/vis absorption spectroscopy to the reaction of $\mathrm{CcmE}$ with apocytochrome $c$ to understand the mechanism of heme transfer and covalent bond formation.

To observe the heme transfer reaction, UV/vis absorption spectra were recorded at every $1 \mathrm{~h}$ intervals after addition of CcmE to apocytochrome $c$. The Soret band of CcmE shifted from 404 to $420 \mathrm{~nm}$ immediately after the reaction, which means the reduction of the heme iron in CcmE. Then, the Soret band gradually diminished with time, but no new bands appeared. This suggests that heme was released from $\mathrm{CcmE}$ despite of the presence of a covalent bond with heme, but not incorporated into apocytochrome $c$. The dissociation of heme from $\mathrm{CcmE}$ was repressed in the absence of apocytochrome $c$. These results indicate that the interaction of apocytochrome $c$ with $\mathrm{CcmE}$ induces a conformational change in $\mathrm{CcmE}$, which facilitates the release of heme and transfers it to apocytochrome $c$. However, the formation of thioether bonds might need other Ccm components.

\section{I-06}

\section{Assembly and transfer of iron-sulfur clusters}

Michael K. Johnson

Department of Chemistry and Center for Metalloenzyme Studies, University of Georgia, Athens, GA 30602, USA.

johnson@chem.uga.edu

Iron-sulfur clusters are one of the most ancient, ubiquitous and structurally diverse classes of biological prosthetic groups. Consequently, $\mathrm{Fe}-\mathrm{S}$ cluster biosynthesis is essential to almost all forms of life and is remarkably conserved in prokaryotic and eukaryotic organisms [1]. Three distinct types of $\mathrm{Fe}-\mathrm{S}$ cluster assembly machinery have been established in bacteria, termed the NIF, ISC and SUF systems, and the ISC and SUF systems form the basis of $\mathrm{Fe}-\mathrm{S}$ cluster biogenesis systems in mitochondria and chloroplasts, respectively. In each case, the overall mechanism involves cysteine desulfurase-mediated assembly of transient clusters on scaffold proteins and subsequent transfer of preformed clusters to apo proteins [2]. A molecular level understanding of the complex processes of $\mathrm{Fe}-$ $\mathrm{S}$ cluster assembly and transfer is now beginning to emerge from the combination of in vivo and in vitro approaches. This overview will highlight recent developments in understanding the mechanism of $[2 \mathrm{Fe}-2 \mathrm{~S}]$ and $[4 \mathrm{Fe}-4 \mathrm{~S}]$ cluster assembly and transfer involving the ubiquitous U-type scaffold proteins and the roles of accessory proteins such as $\mathrm{Nfu}$ proteins and monothiol glutaredoxins in the assembly, storage or transfer of $\mathrm{Fe}-\mathrm{S}$ clusters.

\section{References}

1. Johnson DC, Dean DR, Smith AD, Johnson MK (2005) Annu Rev Biochem 74:247-281

2. Bandyopadhyay S, Chandramouli K, Johnson MK (2008) Biochem Soc Trans 36:1112-1119

\section{I-07}

Partners in regulation of iron homeostasis: Grx3/4 and Fra2 form an unusual [2Fe-2S]-bridged complex Haoran $\mathrm{Li}^{1}$, Nin N. Dingra ${ }^{1}$, Daphne T. Mapolelo ${ }^{2}$, Sunil G. Naik $^{3}$, Greg Keller ${ }^{4}$, Dennis R. Winge ${ }^{4}$, Pamela J. Riggs-Gelasco ${ }^{5}$, Boi Hanh Huynh ${ }^{3}$, Michael K. Johnson ${ }^{2}$, Caryn E. Outten ${ }^{1}$ ${ }^{1}$ Department of Chemistry and Biochemistry, University of South Carolina, Columbia, SC 29208, USA. ${ }^{2}$ Department of Chemistry, University of Georgia, Athens, GA 30602, USA. ${ }^{3}$ Department of Physics, Emory University, Atlanta, GA 30322, USA. ${ }^{4}$ Department of Chemistry and Biochemistry, College of Charleston, Charleston, SC 29424, USA. ${ }^{5}$ Departments of Biochemistry and Medicine, University of Utah, Salt Lake City, UT 84132, USA. caryn.outten@chem.sc.edu The transcription of iron uptake and storage genes in the model eukaryote $S$. cerevisiae is dependent upon mitochondrial Fe-S cluster biosynthesis via a signaling pathway that includes the cytosolic proteins Grx3, Grx4, and Fra2 [1]. We have shown that co-expression of recombinant Fra2 with Grx3 or Grx4 allows purification of a [2Fe$2 \mathrm{~S}^{2+}$-bridged complex. Comparison of the UV-visible, CD, resonance Raman, EPR, EXAFS, and Mössbauer spectra of [2Fe-2S]Grx3/4 vs. [2Fe-2S]-Fra2-Grx3/4 indicates that inclusion of Fra2 in the Grx3/4 Fe-S complex causes a change in the cluster stability and coordination environment. We have also co-expressed and purified mutant forms of Fra2 and Grx3 to identify specific residues that are required for cluster binding and complex formation. Together, these studies suggest that Grx3/4 and Fra2 form a [2Fe-2S]-bridged heterodimer with ligands provided by the active site cysteine of Grx3/4, a histidine in Fra2, and glutathione.

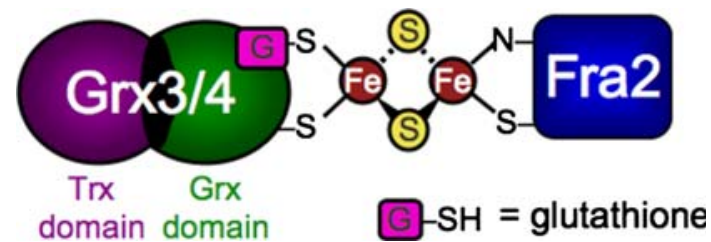

\section{Reference}

1. Kumanovics A, Chen OS, Li L, Bagley D, Adkins EM, Lin H, Dingra NN, Outten CE, Keller G, Winge D, Ward DM, Kaplan J (2008) J Biol Chem 283:10276 


\section{I-08}

The structural basis of the iron-sulfur cluster biosynthesis in plant chloroplasts

Toshiki Yabe ${ }^{1}$, Masaki Unno', Akihiro Kikuchi ${ }^{2}$, Eiki Yamashita $^{3}$, Toshitaka Matsui ${ }^{1}$, Masato Nakai ${ }^{3}$, Masao Ikeda-Saito ${ }^{1}$

${ }^{1}$ Institute of Multidisciplinary Research for Advanced Materials,

Tohoku University, Katahira, Sendai, 980-8577, Japan.

${ }^{2}$ RIKEN Harima Institute, Sayo, Hyogo 679-5148, Japan.

${ }^{3}$ Institute of Protein Research, Osaka University, Suita, 565-0871,

Japan.tyabe@tagen.tohoku.ac.jp

The biosynthesis of iron-sulfur $(\mathrm{Fe}-\mathrm{S})$ clusters is a highly regulated process involving several proteins. Among them, so-called scaffold proteins play pivotal roles in both the assembly and delivery of $\mathrm{Fe}-\mathrm{S}$ clusters. We have focused on $\mathrm{Fe}-\mathrm{S}$ cluster biosynthesis pathway in oxygen-evolving photosynthetic organelles, chloroplasts. $\mathrm{CnfU}$ is evolutionarily highly conserved among oxygen-evolving photosynthetic organisms and has been shown to be important for $\mathrm{Fe}-\mathrm{S}$ cluster biosynthesis in these organisms. We previously demonstrated that the chloroplast $\mathrm{CnfU}$ could actually function as a scaffold for $\mathrm{Fe}-\mathrm{S}$ cluster assembly and delivery [1]. To discuss the $\mathrm{Fe}-\mathrm{S}$ cluster biosynthesis pathway mediated by $\mathrm{CnfU}$ on molecular basis, we have determined the crystal structure of the chloroplast $\mathrm{CnfU}$. In addition, we have succeeded in obtaining information on the structure of $\mathrm{Fe}-\mathrm{S}$ cluster bound on $\mathrm{CnfU}$ by X-ray absorption spectroscopy. Based on the results, we will present the molecular mechanisms of the $\mathrm{Fe}-\mathrm{S}$ cluster transfer mediated by $\mathrm{CnfU}$ on the conference.

References

1. Yabe T, Morimoto K, Kikuchi S, Nishio K, Terashima I, Nakai M (2004) Plant Cell 16:993-1007

2. Yabe T, Yamashita E, Kikuchi A, Morimoto K, Nakagawa A, Tsukihara T, Nakai M (2008) J Mol Biol 381:160-173

\section{I-09}

\section{Insights into assembly of the H-cluster of [FeFe]} hydrogenase

Joan B. Broderick ${ }^{1}$, Eric M. Shepard, Alexandra Bueling, Mark A. Winslow, Shawn E. McGlynn, Neelambari Joshi, John W. Peters

${ }^{1}$ Department of Chemistry and Biochemistry, Montana State University, Bozeman, MT 59717, USA.

jbroderick@chemistry.montana.edu

The $[\mathrm{FeFe}]$ hydrogenase has at its active site an unusual metal cluster, the $\mathrm{H}$ cluster, comprising a $[4 \mathrm{Fe}-4 \mathrm{~S}]$ cubane linked to a $[2 \mathrm{Fe}]$ unit coordinated by cyanide, carbon monoxide, and a unique bridging dithiolate ligand. This bio-organometallic cluster is synthesized in vivo by the action of two radical SAM enzymes (HydE and HydG) and a GTPase (HydF). Evidence suggests that HydF acts as a scaffold for assembly of the H-cluster [1], and that the maturation proteins are responsible for generating and inserting only the $2 \mathrm{Fe}$-subcluster of the H-cluster into HydA (see related talk by John Peters). We will present in this lecture intriguing new findings regarding the role of GTPase activity and radical SAM chemistry in the biosynthesis and assembly of the $\mathrm{H}$-cluster.
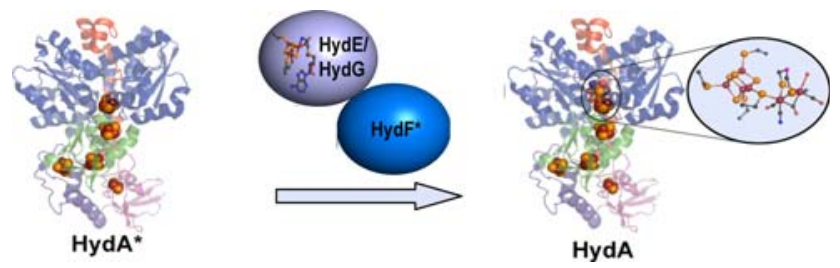

Reference

1. McGlynn SE, Shepard EM, Winslow MA, Naumov AV, Duschene KS, Posewitz MC, Broderick WE, Broderick JB, Peters JW (2008) FEBS Lett 582:2183-2187

\section{I-10}

$\mathrm{CooC}$, the maturation factor of anaerobic CO-dehydrogenase is a nickel binding ATPase

Holger Dobbek, Till Giese, Marlene Grünwald, Jae-Hun Jeoung Laboratory of Bioinorganic Chemistry, University of Bayreuth, 95447 Bayreuth, Germany. Holger.Dobbek@Uni-Bayreuth.de

The maturation of nickel-dependent enzymes requires the participation of accessory proteins. CooC proteins have been implicated in the insertion of nickel into the $\mathrm{Ni}, \mathrm{Fe}$-cluster (cluster $\mathrm{C}$ ) of carbon monoxide dehydrogenases. The ATPase CooC1 from Carboxydothermus hydrogenoformans belongs to the MinD family of SIMIBI type NTPases containing a deviant walker A motif. As-isolated CooC1 is monomeric and shows weak ATPase activity. In the presence of $\mathrm{Ni}(\mathrm{II}), \mathrm{CooC} 1$ dimerizes and binds one $\mathrm{Ni}(\mathrm{II})$ ion per dimer with nanomolar affinity. The crystal structure of $\mathrm{Zn}$-bound $\mathrm{CooC} 1$ shows that a conserved CXC motif constitutes the metal binding site responsible for the dimerization. The presence of ATP and ADP induces dimerization of $\mathrm{CooC} 1$, which is diminished when alanine replaces the signature lysine in the P-loop. The crystal structures of $\mathrm{CooC} 1$ determined in different states together with biochemical data indicate that $\mathrm{CooC} 1$ cycles between an open, metal-bound state and a closed state are capable of ATP hydrolysis in which $\mathrm{CooC} 1$ releases the nickel ion.
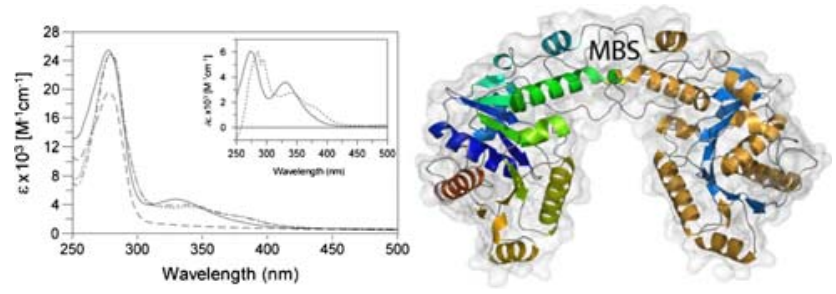

I-11

The role of the Menkes copper-transporting P-type ATPase (ATP7A) in copper homeostasis and regulation of its function

James Camakaris ${ }^{1}$, Nicholas Veldhuis ${ }^{1}$, Ann Gaeth ${ }^{1}$, Nickless Palstra $^{1}$, Adam Southon ${ }^{1}$, Valentina Valova ${ }^{2}$, Phillip Robinson ${ }^{2}$ ${ }^{1}$ Department of Genetics, University of Melbourne, Parkville, VIC 3010, Australia. ${ }^{2}$ Children's Medical Research Institute, Westmead, NSW 2145, Australia. j.camakaris@unimelb.edu.au

The Menkes P-type ATPase (ATP7A) is a transmembrane copper transporter which plays a crucial role in $\mathrm{Cu}$ homeostasis. It transports $\mathrm{Cu}$ across epithelial and endothelial cell barriers such as the gut, kidney, and blood brain barrier. Mutations in ATP7A lead to the often fatal copper deficiency in humans, Menkes disease. ATP7A is also involved in resistance to particular cisplatin cancer chemotherapeutic agents and is likely to play a role in cancer pathophysiology through its involvement in angiogenesis. ATP7A function is largely regulated by its sub-cellular localisation. When $\mathrm{Cu}$ levels are elevated, it traffics from the trans-Golgi network to the plasma membrane and particular endosomes. This is dependent on specific amino acid motifs in ATP7A. Using LC-MS/MS, we have identified kinase phosphorylation sites in cytosolic domains of ATP7A and, importantly, particular sites show Cu-responsive phosphorylation. Mutation studies on these sites have revealed that particular $\mathrm{Cu}$-responsive sites are involved in 
Cu-responsive trafficking of ATP7A. These exciting observations implicate signalling processes in regulation of ATP7A function in various tissues including its responses to $\mathrm{Cu}$ and particular hormones. Using "fluorescence recovery after photobleaching" (FRAP) investigations, we have found that membrane tethering at the plasma membrane appears to be different in polarised compared to non-polarised cells tying in with the physiological function of ATP7A in polarised epithelia. We are investigating ATP7A in retinal pigment epithelial cells and its possible role in serious retinal degeneration diseases. 